\title{
Alcoholismo y ayuda mutua. De la necesidad a la evidencia
}

\section{Alcoholism and mutual help. From necessity to evidence}

\author{
Francisco Pascual Pastor* \\ * Coordinador Médico Unidad de Conductas Adictivas de Alcoi. Colaborador Honorífico Departamento de Biología Aplicada. \\ Universidad Miguel Hernández. Miembro del Instituto de Investigación en Drogodependencias (INID), UMH
}

$\mathrm{E}$ 1 consumo de alcohol es uno de los factores de riesgo más importantes en la salud pública mundial, estando gran parte de los efectos nocivos de su consumo asociados a un deterioro, que puede llegar a ser persistente, a nivel del sistema nervioso central (Soler et al., 2014).

A pesar del paso de los años, los movimientos de ayuda mutua o autoayuda (en ocasiones se utiliza como sinónimo), siguen siendo una realidad en el tratamiento de las adicciones en general y del alcoholismo en particular.

Con una base histórica proveniente de los movimientos de templanza del siglo XIX (Pascual Pastor y Castellano Gómez, 2006) con un carácter incluso religioso o militar en algunos casos y con el objetivo final de conseguir y mantener la abstinencia total a las bebidas alcohólicas, la evolución de estos movimientos ha permitido elaborar programas como el de Alcohólicos Anónimos (AA.AA.) (Pascual Pastor, 2009) basado en los 12 pasos o el de Alcohólicos Rehabilitados (A.R.), con una implicación más multidisciplinar entre Rehabilitados, profesionales (médicos, psicólogos, trabajadores sociales, etc...) y con apoyo familiar a partir del denominado método Minnesota (Carreras Alabau, 2011).
Aunque utilizamos los términos de forma indistinta, la autoayuda y la ayuda mutua, lejos de ser sinónimos son dos términos complementarios, mediante el primero se asumen responsabilidades personales en el cuidado de uno mismo y en el segundo en el apoyo de unos a otros.

En 1978, la Comisión de Salud mental del gobierno de EE.UU, propuso la autoayuda como la principal base de las intervenciones comunitarias en salud mental (Villalba Quesada, 1996). El paso de los años no ha debilitado el modelo, sino que incluso lo ha reforzado, existiendo organizaciones regionales o nacionales en distintos puntos del planeta. Incluso en Europa un colectivo de ellos ha constituido la European Mutual Help Network for Alcohol Related Problems (EMNA), con el objetivo de homogeneizar la atención, ayudar a quien lo precise y hacer advocacy.

En España, a principios del 2015 se ha constituido la Confederación de Adictos Alcohólicos en Rehabilitación y Familiares de España (CAARFE), que recogiendo el testigo de la extinta Federación de Alcohólicos Rehabilitados de España (FARE), ha completado la estructura con la participación de tres pilares, los enfermos en rehabilitación, familiares y profesionales, e incluyendo otras adicciones distintas al 
alcohol, ya que las formas de consumo han cambiado y el perfil del consumidor presenta un patrón de policonsumo o multiadicción, normalmente de alcohol conjuntamente con cocaína, cannabis, tabaco o incluso adicciones comportamentales, especialmente la ludopatía.

Llegados a este punto vamos a intentar definir estos colectivos o movimientos, puntualizar sus objetivos y valorar su eficacia a partir de la evidencia científica.

Los Grupos de Ayuda Mutua (GAM), son una modelo de salud, con especial relevancia del modelo de "iguales", formado por personas que sufren la misma patología o conflicto. Aparecieron como respuesta a un vacío de los servicios profesionales, pero pronto se vio su eficacia y la propia OMS lo recomendó para ciertas necesidades socio sanitarias (OMS, 1987).

Sus fortalezas se basan en el aprendizaje, el apoyo emocional, la participación y la autoestima, el poder solicitar cambios sociales y la capacidad para organizarse.

Sus bases principales son las reuniones, conocimiento y experiencia personal y el apoyo entre sus participantes, originariamente sin profesionales, pero a tenor de la evolución de los mismos se vio su validez para la comunidad que se veía incrementada con la participación de profesionales para completar el tratamiento (Roca Soriano. 1998), con la propia reciprocidad de este tipo de enfoque terapéutico basado en Dar - Recibir y Devolver (Módena, 2009).

Se trata de una estructura fundamental y esencialmente democrática, adaptada al lugar, el ambiente y la cultura donde se comparten experiencias sin generalizar, sin consejos, sin juicios de valor y sin hablar desde el saber ni desde el ser (Montaño Fraire, 2010).

Cuyos objetivos son fundamentalmente, satisfacer una necesidad común, superar un obstáculo que perturba seriamente la vida y conseguir los cambios sociales o personales deseados (Villalba Quesada, 1996), en el caso de las adicciones sería alcanzar y mantener la abstinencia, trabajar los propios hándicaps y cambiar la forma de ser, de actuar y de relacionarse.

Según García Roldán et al. (1997), en la interacción que se produce en los grupos de ayuda mutua, los pacientes adquieren conocimientos sobre su enfermedad, desmienten mitos sobre las drogas, aprenden recursos de enfrentamiento y sobre todo comparten vivencias sobre cómo enfrentar y superar los conflictos que le ha generado su adicción, su consumo de alcohol, y como lograr cambiar su estilo de vida y mantenerse en abstinencia, insertándose en la vida familiar, laboral y social (Martínez Leiva et al, 2010).

Pero, ¿realmente son efectivos?

Pues según distintos estudios parece ser que sí, ya que en 2004 se realizó un estudio con 279 pacientes dependientes al alcohol (Zemore et al, 2004), con buenos resultados, analizando la evolución de los pacientes que seguían el programa de los 12 pasos, lo que fue corroborado igualmente por la valoración del proyecto Match (Pagano et al, 2004), llegando a aseverar que los que participaban en los GAM mantenían la abstinencia durante más tiempo que los que seguían un tratamiento convencional, por lo que se alentaba a los facultativos a remitir a los pacientes alcohólicos a estos grupos.

Estos resultados llevaron a la American Psychiatric Association en 2006 a situar en un primer nivel de recomendación los GAM para el tratamiento del alcoholismo (APA, 2006)

No obstante, la revisión Cochrane basada fundamentalmente en el trabajo hecho por AA.AA. no es concluyente, y se señalan la falta de estudios más completos para constatar la evidencia científica (Ferri et al., 2006).

Paradójicamente, el modelo de AA.AA. es el de mayor difusión y antigüedad en el mundo moderno, el único requisito es querer dejar de consumir y es que, aunque el grupo sea heterogéneo, hay un objetivo común, la abstinencia, aunque la asistencia para obtener buenos resultados debe ser al menos 1 vez por semana (Martínez Ortiz, 2013). Otros estudios señalen que acudir dos días a la semana produce al menos 3 días más de abstinencia de alcohol al mes (Humphreys et al., 2014).

En España se están empezando a valorar resultados. En 2013 se publicó un estudio basado en la integración de los GAM para familiares en un programa público de tratamiento del alcoholismo (Rubio et al., 2013), que concluía que las intervenciones con familiares dependientes del alcohol han demostrado su eficacia para mejorar el pronóstico de la dependencia alcohólica, la cual mejora cuando estos familiares acuden a los GAM, obteniendo en los propios pacientes menos abandonos y menos días de consumo durante el tiempo de tratamiento.

La coordinación con el sistema sanitario y la pertenencia a estos grupos, incrementa la abstinencia y disminuye el número de recaídas, comprobando que a mayor tiempo de asistencia a estos grupos menor posibilidad de sufrir una recaída en el consumo (Pascual, 2015), convirtiendo los GAM en una buena estrategia terapéutica unida a la asistencia pública para los problemas relacionados con el alcohol.

Según la Guía Clínica de Socidrogalcohol la evidencia para estas intervenciones tiene un nivel de $2 \mathrm{~B}$ y un grado de recomendación B (Tomás, 2013)

Así está claro que se trata de estrategias terapéuticas útiles, pero se precisa una evaluación más exhaustiva y una homogenización de recursos y métodos para poder decir claramente que se trata de una buena opción terapéutica, de momento lo que sí que está claro es que estamos delante de un complemento terapéutico útil para disminuir las recaídas, incrementar la adherencia al tratamiento y mantener la abstinencia a más largo plazo. Con mejores resultados cuando se trabaja con los familiares y enfermos al mismo tiempo y con el correspondiente respaldo y tutela profesional.

Estamos pues delante de un modelo de atención a los problemas relacionados con el consumo de bebidas alcohólicas y de otras adicciones, que puede retomarse como comple- 
mento ideal para acercar el tratamiento a pacientes y familiares, por cercanía geográfica, facilidad horaria y empatía de la propia estructura formada por personas que han pasado por la misma adicción, pero debemos insistir con la participación de profesionales y coordinado con los servicios socio-sanitarios especializados en este tipo de patologías.

\section{Bibliografía}

American Psychiatric Association. (2006). Practice guideline for the treatment of patients with substance use disorders, second edition. Washington, DC: American Psychiatric Association.

Carreras Alabau, A. (2011). Intervenciones en el Consumo de Alcohol: De los Grupos de Autoayuda a la Regulación de la Propia Conducta. ¿Métodos Complementarios o Antagónicos? Clínica Contemporánea, 2, 249-269.

Ferri, M., Amato, L. y Davoli, M. (2006). Alcoholics Anonymous and other 12-step programmes for alcohol dependence. Cochrane Database of Systematic Reviews, 3. Art. No.: CD005032. DOI: 10.1002/14651858.CD005032.pub2.

García Roldán, R., Garcés Rodríguez, A. Z., López Martínez, M. y López, E. F. (1997). Caracterización sociofamiliar en un grupo de pacientes alcohólicos. Revista Cubana de Medicina General Integal, 13, 139-142.

Humphreys, K., Blodgett, J. C. y Wagner, T. H. (2014). Estimating the efficacy of Alcoholics Anonymous without self-selection bias: an instrumental variables re-analysis of randomized clinical trials. See comment in PubMed Commons belowAlcoholism: Clinical and Experimental Research, 38, 2688-2694. doi: 10.1111/ acer. 12557.

Martínez Leiva, A., Castillo Rojas, L., Guevara Sanabria, M., Gutierrez Villa, N. y Perdomo Gómez, A. (2010). Caracterización socio-psicológica de los pacientes del grupo de ayuda mutua de alcoholismo en el municipio Morón. MEDICIEGO, 16, Supl. 1.

Martínez Ortiz, E. (2013). Tratamientos de autoayuda y grupos de 12 pasos. En: E. Velázquez, G. Castaño. (Coord.). Adicciones: aspectos clínicos y psicosociales, tratamiento y prevención. Medellín: Editorial CIB.

Módena, M. A. (2009). Alcoholismo, ayuda mutua y autoayuda. Desacatos, 29, 7-10.

Montaño Fraire, R. (2010). RedCAPA. Reseña y análisis de un sistema de ayuda mutua para adicciones en Madrid, España. Actas del XI Congreso Nacional de Especialistas en Adicciones Ciudad de México, Distrito Federal.

Organización Mundial de la salud (1987). Conference International Information Centre on Self-Help and Health. p. 33-37.

Pagano, M. E., Friend, K. B., Tonigan, J. S. y Stout, R. L. (2004). Helping other alcoholics in alcoholics anonymous and drinking outcomes: findings from project MATCH. Journal of Studies on Alcohol, 65, 766-773.
Pascual Pastor, F. (2009). La Autoayuda en drogodependencias en el siglo XXI. Revista Española de Drogodependencias, 34, 124-127.

Pascual Pastor, F. y Castellano Gómez, M. (2006). Grupos de Autoayuda. En: J. Pérez de los Cobos, J.C. Valderrama Zurián, G. Cervera Martínez, G. Rubio Valladolid (Direct.). Tratado SET de Trastornos adictivos (pp. 450454) Madrid: Editorial Médica Panamericana.

Pascual, F. (2015). The rol of mutual-help for alcohol related problems in Spain. Alcohol and Alcoholism, 50, i23.

Roca Soriano, F. (1998). Los "desconocidos" grupos de ayuda mutua. Cuadernos de Trabajo Social, 11, 251-263.

Rubio, G., Martínez Gras, I., Ponce Alfaro, G., Quinto Guillén, R., Jurado Barba, R. y Jiménez Arriero, M. A. (2013). Integración de los grupos de ayuda muta para familiares en un programa público de tratamiento del alcoholismo. Adicciones, 25, 37-44.

Soler Gonzaléz, C., Balcells Oliveró, M. y Gual Solé, A. (2014). Alcohol related brain damage. State of the art and call for action. Adicciones, 26, 199-207.

Tomás, V. (2013). Intervención bio-psico-social para los tratamientos relacionados con el consumo de alcohol. En: Alcoholismo. Guías Clinicas Socidrogalcohol basadas en la evidencia científica (pp. 261) Barcelona: Socidrogalcohol.

Villaba Quesada, C. (1996). Los grupos de apoyo basados en la autoayuda. Una propuesta para el inicio del acompañamiento profesional. Psychosocial Intervention, 5, 23-42.

Zemore, S. E., Kaskutas, L.A. y Ammon, L. N. (2004). In 12-step groups, helping helps the helper. Addiction, 99, 1015-1023. 